\title{
Vertical level selection for temperature and trace gas profile retrievals using IASI
}

\author{
R. A. Vincent ${ }^{1,2}$, A Dudhia ${ }^{1}$, and L. J. Ventress ${ }^{1}$ \\ ${ }^{1}$ Atmospheric, Oceanic and Planetary Physics, Clarendon Laboratory, Parks Road, Oxford, OX1 3PU, UK \\ ${ }^{2}$ Air Force Institute of Technology, Wright-Patterson Air Force Base, Ohio, 45433, USA \\ Correspondence to: R. A. Vincent (vincent@atm.ox.ac.uk)
}

Received: 30 January 2015 - Published in Atmos. Meas. Tech. Discuss.: 9 March 2015

Revised: 6 May 2015 - Accepted: 17 May 2015 - Published: 8 June 2015

\begin{abstract}
This work presents a new iterative method for optimally selecting a vertical retrieval grid based on the location of the information while accounting for inter-level correlations. Sample atmospheres initially created to parametrise the Radiative Transfer Model for the Television Infrared Observation Satellite Operational Vertical Sounder (RTTOV) forward model are used to compare the presented iterative selection method with two other common approaches, which are using levels of equal vertical spacing and selecting levels based on the cumulative trace of the averaging kernel matrix (AKM). This new method is shown to outperform compared methods for simulated profile retrievals of temperature, $\mathrm{H}_{2} \mathrm{O}, \mathrm{O}_{3}, \mathrm{CH}_{4}$, and $\mathrm{CO}$ with the Infrared Atmospheric Sounding Interferometer (IASI). However, the benefits of using the more complicated iterative approach compared to the simpler cumulative trace method are slight and may not justify the added effort for the cases studied, but may be useful in other scenarios where temperature and trace gases have strong vertical gradients with significant estimate sensitivity. Furthermore, comparing retrievals using a globally optimised static grid vs. a locally adapted one shows that a static grid performs nearly as well for retrievals of $\mathrm{O}_{3}, \mathrm{CH}_{4}$, and CO. However, developers of temperature and $\mathrm{H}_{2} \mathrm{O}$ retrieval schemes may at least consider using adaptive or location specific vertical retrieval grids.
\end{abstract}

\section{Introduction}

Retrieved profiles of temperature and composition from nadir-viewing instruments are often presented on a grid much finer than can be justified given the actual vertical resolution of the measurements. Therefore, we propose a method to determine the optimal subset of vertical levels from a fine vertical grid by selecting levels according to their contribution to the degrees of freedom that come from the signal (DFS) rather than the a priori (Rodgers, 2000).

When designing a retrieval scheme, it is useful first to determine the subset of coarse vertical levels that efficiently contribute to the estimate. By reducing the number of attempted estimates, the retrieval relies less on formal prior knowledge and becomes more sensitive to the true state. There are also computational benefits during the retrieval due to the improved conditioning of the problem, possibly faster convergence and greater tolerance of ad hoc assumptions in the a priori.

Consider, for example, the Infrared Atmospheric Sounding Interferometer (IASI) level two (L2) product; where temperature, water vapour, and ozone profiles are presented on a vertical grid of 100 pressure levels ranging from surface pressure up to $0.016 \mathrm{hPa}$, an altitude of approximately $80 \mathrm{~km}$ (August et al., 2012). While vertical resolution at this scale is highly desirable, retrievals can only be performed on such fine grid spacing at the expense of heavy dependence on the a priori estimate or other constraints.

Post-processing methods are developed to reduce the reliance upon a priori information. Possible a priori sources include Numerical Weather Prediction (NWP) data and chemical transport models such as the Goddard Earth Observing System Chemical transport model (GEOS-Chem; Bey et al., 2001). While similar sources are generally of high quality, modelling artefacts do appear in atmospheric retrievals. For example, Bowman et al. (2006) recognised during the development of the Tropospheric Emission Spectrometer (TES) 
retrieval method that the full state vector grid (67 levels) used in calculating radiative transfer may be too fine for the purposes of a retrieval. They therefore decided upon a coarser retrieval grid of 14 pressure levels. However, since the DFS in their methane retrieval ranged from 0.5 to 2.0 (Payne et al., 2009), the subsequent grid was still too fine for methane and discontinuities in volume mixing ratio (VMR) were observed (Brasseur et al., 1998). To improve the TES results, Payne et al. (2009) remapped the estimates to a single "representative tropospheric VMR" level that effectively removed a priori artefacts from the retrieval.

Previous work by von Clarmann and Grabowski (2007); Ceccherini et al. (2009), and Joiner and Da Silva (1998) show that post-processing can transform a regularised retrieval to a maximum likelihood estimate of the atmospheric state. The two ideas of using a coarse retrieval grid to constrain the solution set and reducing a priori in the estimate can be combined and implemented during the retrieval algorithm, while minimising the loss of information.

The presented work is applied to profile retrievals of temperature, $\mathrm{H}_{2} \mathrm{O}, \mathrm{O}_{3}, \mathrm{CH}_{4}$, and $\mathrm{CO}$ using IASI. However, this methodology can be readily applied to other infrared atmospheric nadir sounding instruments, e.g. TES, Atmospheric InfraRed Sounder (AIRS; Aumann et al., 2003), and Crosstrack Infrared Sounder (CrIS; Han et al., 2013) as well as different species. Comparisons between the new vertical selection method and two other common methods are presented.

Section 2 outlines the theoretical basics of optimal estimation and the constraint mapping process necessary to understand vertical grid selection. Section 3 describes the proposed vertical selection method as well as the two simpler alternative methods. Available methods are analysed and compared in Sect. 4, while Sect. 5 discusses the trade-off between using a globally constructed vertical grid vs. atmosphere specific grids. Finally, conclusions are summarised in Sect. 6.

\section{Theoretical background}

Atmospheric profile retrievals with a nadir viewing satellite tend to be significantly ill-conditioned. In other words, the attempted number of estimated parameters $(n)$ is greater than the DFS. Therefore, constraints must be applied to stabilise the retrieval. Constraints in vector and matrix form can be chosen in a variety of ways (Kulawik et al., 2006). When constraints are applied to an ill-conditioned problem, the a priori information inevitably becomes an artefact of the resulting estimate (Rodgers, 2000). At this point the designer of the retrieval has two choices, (1) tolerate artefacts from the a priori or (2) move to a representation that is better conditioned.

This section reviews inverse theory, applied to illconditioned atmospheric sounding. While there are at least two separate notations commonly used, we adopt the notation consistent with Rodgers (2000), where numerous derivations supporting the following discussion can be found.

\subsection{Optimal estimation}

When the radiative transfer function is sufficiently linear about a reference state vector $\left(\boldsymbol{x}_{\mathbf{0}}\right)$ of length $n$, the forward model $(F)$ can be linearised according to

$\boldsymbol{y}-F\left(x_{0}\right)=\mathbf{K}\left(\boldsymbol{x}-\boldsymbol{x}_{\mathbf{0}}\right)+\boldsymbol{\epsilon}$,

where $\boldsymbol{y}$ is the measured spectrum of length $m, \boldsymbol{x}$ is the true state to be estimated, and $\boldsymbol{\epsilon}$ is the error in the measured signal relative to the forward model. Furthermore, $\mathbf{K} \in \mathbb{R}^{m \times n}$, referred to as the Jacobian matrix, is defined to be a matrix of partial derivatives such that $K_{i j}=\partial F_{i}(\boldsymbol{x}) / \partial x_{j}$.

Solutions to Eq. (1) can be estimated in the maximum a posteriori framework (a.k.a. optimal estimation) by considering a linearisation about an a priori reference state $\left(\boldsymbol{x}_{\mathrm{a}}\right)$. Estimates of an atmospheric state $(\hat{\boldsymbol{x}})$ are given by

$$
\begin{aligned}
\hat{\boldsymbol{x}} & =\boldsymbol{x}_{\mathrm{a}}+\left(\mathbf{K}^{\mathrm{T}} \mathbf{S}_{\epsilon}^{-1} \mathbf{K}+\mathbf{S}_{\mathrm{a}}^{-1}\right)^{-1} \mathbf{K}^{\mathrm{T}} \mathbf{S}_{\epsilon}^{-1}\left(\boldsymbol{y}-F\left(\boldsymbol{x}_{\mathrm{a}}\right)\right) \\
& =\boldsymbol{x}_{\mathrm{a}}+\mathbf{G}\left(\boldsymbol{y}-F\left(\boldsymbol{x}_{\mathrm{a}}\right)\right),
\end{aligned}
$$

where $\mathbf{G}$ is referred to as the gain matrix. The covariance matrix of the stochastic error in the measurements is denoted as $\mathbf{S}_{\epsilon}$. Since raw spectra from a Fourier transform spectrometer (FTS) such as IASI are generally uncorrelated, $\mathbf{S}_{\epsilon}$ has zeroes in the off-diagonal elements while the diagonal elements are the variances of the signal at that spectral position. However, because IASI spectra are apodized (Amato et al., 1998), offdiagonal spectral correlations are thus introduced into $\mathbf{S}_{\epsilon}$.

The term a priori is meant to include both a mean state, $\boldsymbol{x}_{\mathrm{a}}$, and its covariance, $\mathbf{S}_{\mathrm{a}}$. Inverting $\mathbf{S}_{\mathrm{a}}$ in Eq. (2) applies a "soft" constraint upon the solution, penalising estimates that deviate greatly from the profile provided in the prior estimate. One method to determine $\mathbf{S}_{\mathrm{a}}$ for atmospheric temperature and trace gases is to download analysis data from a NWP source and calculate statistical covariances from a global ensemble or about the local region considered. However, statistical covariances calculated this way may not be invertible if the ensemble does not contain enough truly independent sample atmospheres. In this case, $\mathbf{S}_{\mathrm{a}}^{-1}$ might be replaced with an alternative method, such as Twomey-Tikhonov regularisation, where smoothness constraints are imposed by considering first and second derivatives of the profile and treated as tuning parameters (Kulawik et al., 2006). While such methods are common, they include less prior knowledge in the sense that higher-order physical correlations are intentionally ignored and suggest that the dimensionality of the retrieval should be reduced to improve the condition of the inverse problem.

Diagnostic information about the retrieval is succinctly contained in a unitless $n \times n$ matrix known as the averaging kernel matrix (AKM), defined as

$\mathbf{A}=\mathbf{G K}=\frac{\partial \hat{\boldsymbol{x}}}{\partial \boldsymbol{x}}$. 
In this case, the rows of $\mathbf{A}$ correspond to the retrieved profile levels and can be thought of as smoothing functions ideally peaking at the referenced level, but with finite widths providing a measure of vertical resolution. Columns of $\mathbf{A}$ depict the response of the retrieval to $\delta$-function perturbations in the true state profile levels. Furthermore, Eq. (2) can be rewritten in the more insightful but less practical form,

$\hat{\boldsymbol{x}}=\left(\mathbf{I}_{n}-\mathbf{A}\right) \boldsymbol{x}_{\mathrm{a}}+\mathbf{A} \boldsymbol{x}+\mathbf{G} \boldsymbol{\epsilon}$,

where $\mathbf{I}_{n}$ is the identity matrix with $n$ diagonal elements. Written this way, it becomes clear that the estimate of state, $\hat{\boldsymbol{x}}$, is a weighted average of the true state and the prior state. Ideally, A approaches the identity matrix and no prior state appears in the estimate. However, this is seldom the case for nadir viewing unless performing a maximum likelihood retrieval where there is by definition no a priori information.

Repeated analysis of $\mathbf{A}$ can be unwieldy when developing a retrieval algorithm. Therefore, a scalar "figure of merit" is often desirable that allows for multiple matrices of $\mathbf{A}$ to be compared in a straightforward manner. The DFS is one such possible metric and is calculated by taking the trace of the averaging kernel matrix,

$d_{\mathrm{s}}=\operatorname{Tr}(\mathbf{A})$.

Perfectly conditioned inverse problems will have DFS values equal to the number of state parameters, $n$.

\subsection{Constraint mapping}

Solutions to ill-conditioned inverse problems can be improved by simply reducing the number of estimated parameters. However, information content may be lost if the retrieved state is reduced too much. Therefore, as the original parameter space is reduced, the information content should be monitored in a consistent mathematical way. This is done by defining operators that map the retrieval between the original and reduced state space.

Consider two vertical grids for the problem of retrieving atmospheric profiles. First, a fine grid from the discretisation of the full state vector, $\boldsymbol{x}$, chosen with enough vertical resolution to accurately calculate the equations of radiative transfer. Second, a coarser grid on which the retrieval is carried out, here on referred to as the retrieval grid $\left(z \in \mathbb{R}^{l}\right)$, where $l<n$. A coarser retrieval grid is necessary when the DFS are significantly less than $n$, in order to improve the condition of the retrieval. Mapping from the fine to the coarse grid imposes a "hard" constraint, where only solutions to the reduced representation are considered.

For convenience we apply a linear mapping from the retrieval to the fine grid so that

$\boldsymbol{x}=\mathbf{W} z$.

Here $\mathbf{W} \in \mathbb{R}^{n \times l}$ is a mapping matrix that is commonly a piecewise linear interpolation operator. In fact, $\mathbf{W}$ could be any general linear transformation that maps the full state vector to a reduced retrieval vector, such as a truncated right singular vector matrix of $\mathbf{A}$ or the signal to noise matrix (Ceccherini et al., 2009; Rodgers, 2000). While singular value decomposition methods guarantee maximum retention of DFS, they transfer the full state vector into a reduced space that has no direct physical meaning. Here we apply linear interpolation to maintain a physical link between elements of the state vector and levels in the atmosphere.

To transform the a priori to the retrieval state space, the following averaging operation is required,

$z=\mathbf{W}^{*} \boldsymbol{x}$

where $\mathbf{W}^{*}$ is the pseudo inverse of $\mathbf{W}$. While there are infinite ways in which $\mathbf{W}^{*} \mathbf{W}=\mathbf{I}_{l}$, the most common is to define $\mathbf{W}^{*}$ in the least-squares sense,

$\mathbf{W}^{*}=\left(\mathbf{W}^{\mathrm{T}} \mathbf{W}\right)^{-1} \mathbf{W}^{\mathrm{T}}$.

With these operators, any number of mathematical transformations are possible that interpolate or average parameters between the fine and coarse grids. However, care must be taken that such operations have sound physical reasoning. For example, the prior covariance of the retrieval can be expressed on the coarse retrieval grid

$\mathbf{S}_{z \mathrm{a}}=\mathbf{W}^{*} \mathbf{S}_{\mathrm{a}} \mathbf{W}^{* \mathrm{~T}}$,

but interpolating $\mathbf{S}_{z \mathrm{a}}$ to a finer grid cannot be assumed valid if the prior covariance is only known on the coarse grid. This is because off-diagonal elements in $\mathbf{S}_{\mathrm{a}}$ represent physical correlations between various pressure levels and may not follow Gaussian statistics (von Clarmann, 2014).

More rigorous derivations and discussions of mapping between states can be found in Worden et al. (2006), Bowman et al. (2006), and Rodgers (2000, ch. 10). Summarising the key mapping relationships relevant to this application, the following list is helpful:

$$
\begin{aligned}
& \{m \times l\} \mathbf{K}_{z}=\mathbf{K}_{x} \mathbf{W}, \\
& \{l \times m\} \mathbf{G}_{z}=\left(\mathbf{K}_{z}^{\mathrm{T}} \mathbf{S}_{\epsilon}^{-1} \mathbf{K}_{z}+\mathbf{S}_{z \mathrm{a}}^{-1}\right)^{-1} \mathbf{K}_{z}^{\mathrm{T}} \mathbf{S}_{\epsilon}^{-1}, \\
& \{n \times n\} \mathbf{A}_{x}=\mathbf{W G}_{z} \mathbf{K}_{x}=\mathbf{G}_{x} \mathbf{K}_{x}, \\
& \{l \times l\} \mathbf{A}_{z}=\mathbf{G}_{z} \mathbf{K}_{x} \mathbf{W}=\mathbf{W}^{*} \mathbf{A}_{x} \mathbf{W} .
\end{aligned}
$$

While the retrieval is performed on the coarse grid, the resulting atmospheric profile is interpolated to the fine grid providing the final estimate. Therefore, when calculating the DFS in Eq. (5) we must use the true averaging kernel, $\mathbf{A}_{x}$, and not $\mathbf{A}_{z}$. Alternatively, one could also retrieve coarse perturbations to a fine grid profile $\left(\boldsymbol{x}_{\mathbf{0}}\right)$ to ensure sharp features, such as the tropopause, were maintained. In which case, these mapping relationships still hold true. 


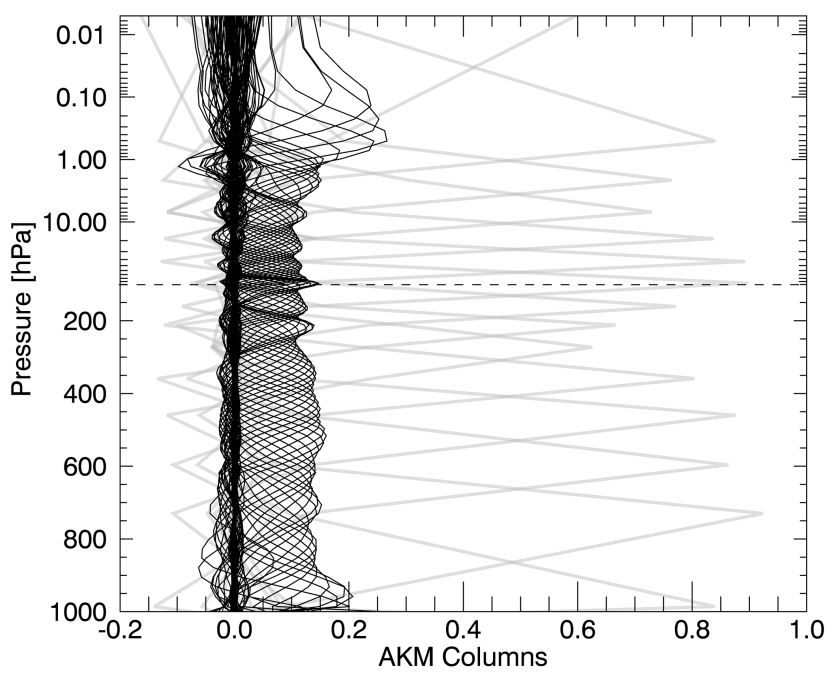

Figure 1. AKM columns are plotted for a sample temperature retrieval with IASI using a fine vertical grid (black) compared to a coarser grid (grey) selected using the iterative vertical selection method. Note that the pressure axis changes from linear to logarithmic above $100 \mathrm{hPa}$ (dashed line).

\section{Profile level selection}

From inspection of Eqs. (11) and (12), selecting an appropriate vertical grid depends upon three things; (1) the radiative response of the atmosphere, (2) the spectral resolution and noise characteristics of the observation instrument, in this case IASI, and (3) the chosen prior covariance matrix, $\mathbf{S}_{\mathrm{a}}$. Since IASI is already designed and collecting data, the only free parameter is the prior covariance. Therefore, it is important to consider that $\mathbf{S}_{\mathrm{a}}$ should be decided upon before attempting to optimise a retrieval grid.

When determining a coarse retrieval grid the number and location of profile levels can be chosen in an ad hoc manner or decided based upon the distribution of information in the profile. The DFS is a natural scalar metric of information to use when constructing and comparing different vertical grids, because it can be directly compared to the number of attempted retrieval levels. When the DFS is approximately equal to the number of levels, then little prior knowledge appears in the estimate. Other possible scalar metrics of information include the Shannon information content and the trace of the Fisher information matrix (Rodgers, 2000, ch. 2). For this particular application the DFS is the most appropriate. With this in mind, three vertical level selection methods are described, ranging from the simple to the complex.

\subsection{Equal spacing}

The simplest possible selection method is to segment the atmosphere into layers of equal thickness. Levels of equal pressure may be used for better tropospheric sensitivity, or levels of equal height for stratospheric sensitivity. Nadir-viewing

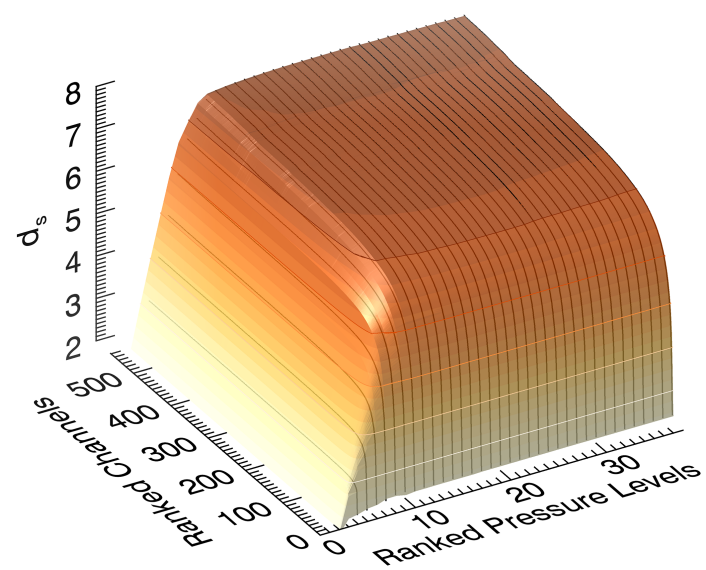

Figure 2. DFS for a temperature retrieval with IASI vs. both ranked atmospheric pressure levels and ranked spectral channels from the $\mathrm{CO}_{2}$ spectrum. The black lines show contours of number of pressure levels and the coloured highlight lines show contours of integer DFS.

instruments such as IASI are typically more sensitive to the troposphere, so equal pressure spacing will be assumed for these comparisons, even though it is clearly inappropriate for species with stratospheric concentrations such as ozone.

\subsection{Cumulative trace}

Alternatively, a vertical selection method proposed by von Clarmann and Grabowski (2007) originally for removing hidden a priori from retrieved estimates is commonly used (Payne et al., 2009). This method utilises the vertical distribution of the DFS through the profile on the fine grid. From the averaging kernel matrix, $\mathbf{A}$, the cumulative trace is calculated as a function of the vertical axis, showing the contribution of each level to the total DFS of the retrieval. Next, the cumulative trace is segmented into equal spacings of number $l$ and the selected coarse vertical levels interpolated from this curve. The resulting coarse pressure grid is thus irregularly spaced based on the vertical density of the DFS.

While using the diagonal of $\mathbf{A}$ is a clear improvement over equal pressure spacings, ignoring the off-diagonal sensitivities is a concern. This is because the original AKM expressed on the fine grid is likely to change morphology as levels are combined; hence the impact on the diagonal will not generally be the simple cumulation assumed. As a result, the vertical partitioning of DFS for a given atmospheric profile retrieved over, for example, 100 levels may differ from a 10 level retrieval due to correlations between levels introduced both spectrally and with $\mathbf{S}_{\mathrm{a}}$. Thus, a vertical selection method is desired that accounts for off-diagonal changes in $\mathbf{A}_{x}$ during the selection process. 


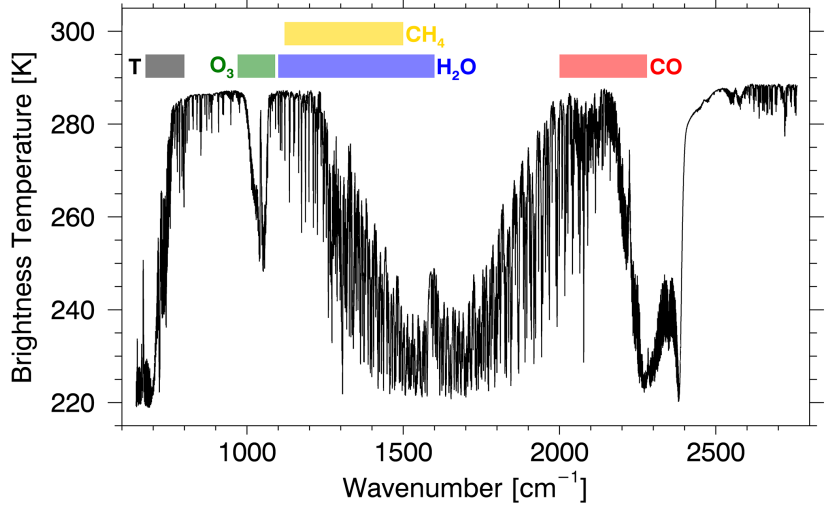

Figure 3. Simulated IASI spectrum showing the spectral ranges considered in this study, which are typical for temperature and trace gas profile retrievals with this instrument.

\subsection{Iterative selection}

The proposed vertical selection method is outlined as follows.

1. Calculate the DFS on the fine grid by making $\mathbf{W}=\mathbf{I}_{n}$. Doing so sets the coarse grid equal to the fine grid.

2. Next, a single level is removed by modifying $\mathbf{W}$ in accordance with the chosen interpolating method. In this case, the piecewise linear method is used for simplicity.

3. The resulting DFS from removing that level are determined from Eqs. (12) and (5). Each possible level is removed individually and its effect on the DFS noted. The removed level that decreased the DFS the least is then discarded. Removed levels are merged into neighbouring levels by redefining the $\mathbf{W}$ matrix.

4. This process is repeated to find the second-least important level until all vertical levels have been ranked and discarded down to the two levels that contribute the most to the DFS.

Following this method results in a ranking of the vertical levels on the fine grid that can be truncated to produce an optimal representation on the retrieval grid for any number of levels.

To visualise the effect, Fig. 1 shows the columns of two AKMs for a temperature retrieval with IASI using part of the $\mathrm{CO}_{2}$ spectral feature between 675 and $800 \mathrm{~cm}^{-1}$. The smaller amplitude responses are for a retrieval on a fine 100 level pressure grid. While the larger more peaked responses are for a 15 level grid $\left(d_{\mathrm{s}} \approx 12\right)$ chosen using this iterative selection method. Figure 1 highlights a fundamental trade-off inherent to constrained retrievals with fixed information content: more parameters can be retrieved with less sensitivity to the true state or fewer estimates attempted with greater sensitivity to the true state.

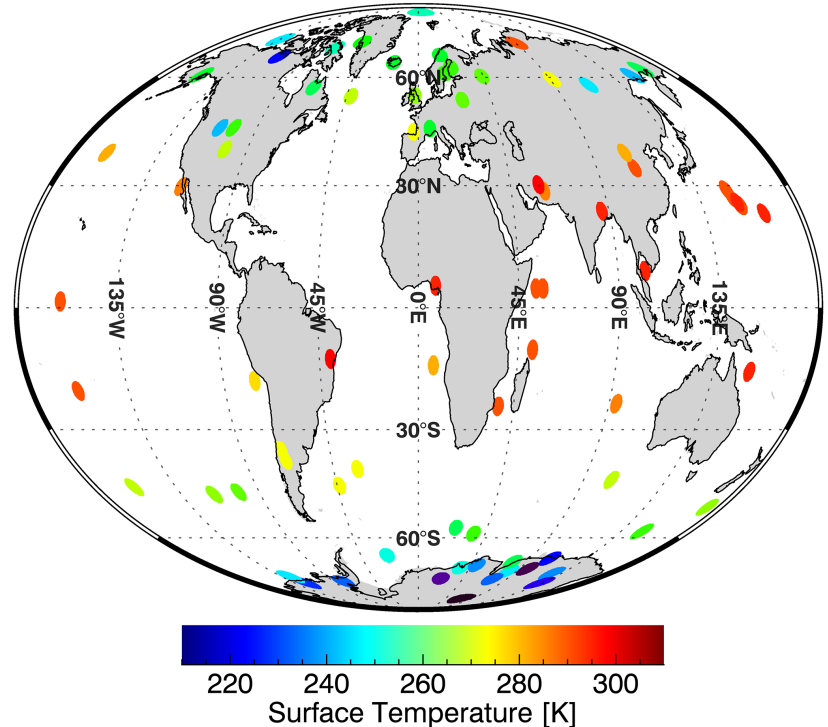

Figure 4. Sampling locations of the 80 atmospheres comprising the RTTOV training ensemble colour coded according to the atmospheric surface temperatures of the profiles.

Clearly, the downside to this iterative selection method is the added complexity and computational cost of checking each available vertical level during the merging process, as compared to the method of interpolating from the cumulative trace of A. However, the iterative steps may be necessary to maximise DFS when there are significant off-diagonal sensitivities in A. In the absence of off-diagonal sensitivities, the two methods converge on the same coarse vertical grid.

\subsection{Deciding upon the number of levels}

Choosing the number of retrieval levels ultimately depends upon the tolerance for a priori appearing in the estimate. For an effectively a priori free retrieval, the number of estimates should be at most equal to the DFS on the fine grid rounded down (von Clarmann and Grabowski, 2007), but this comes at the cost of a modest reduction in DFS. However, if it is acceptable for some a priori to appear in the estimate, then how many levels beyond the DFS should be included?

Figure 2 helps shed some light upon this question. The surface plot shown is also for a temperature retrieval with IASI using the $\mathrm{CO}_{2}$ region previously mentioned. Pressure levels have been ranked by the iterative method just described and spectral channels have been ranked according to the channel selection method found in Ventress and Dudhia (2013). The vertical and spectral selection methods bear much similarity. For this particular constrained retrieval, $d_{\mathrm{s}}=7.6$ on a fine vertical grid.

Notice that the DFS increases nearly linearly with pressure level number initially, but quickly reaches a plateau once $l>d_{\mathrm{s}}$. The DFS axis begins at $d_{\mathrm{s}}=1.99$, where retrieving temperature at two large bulk layers (troposphere and strato- 

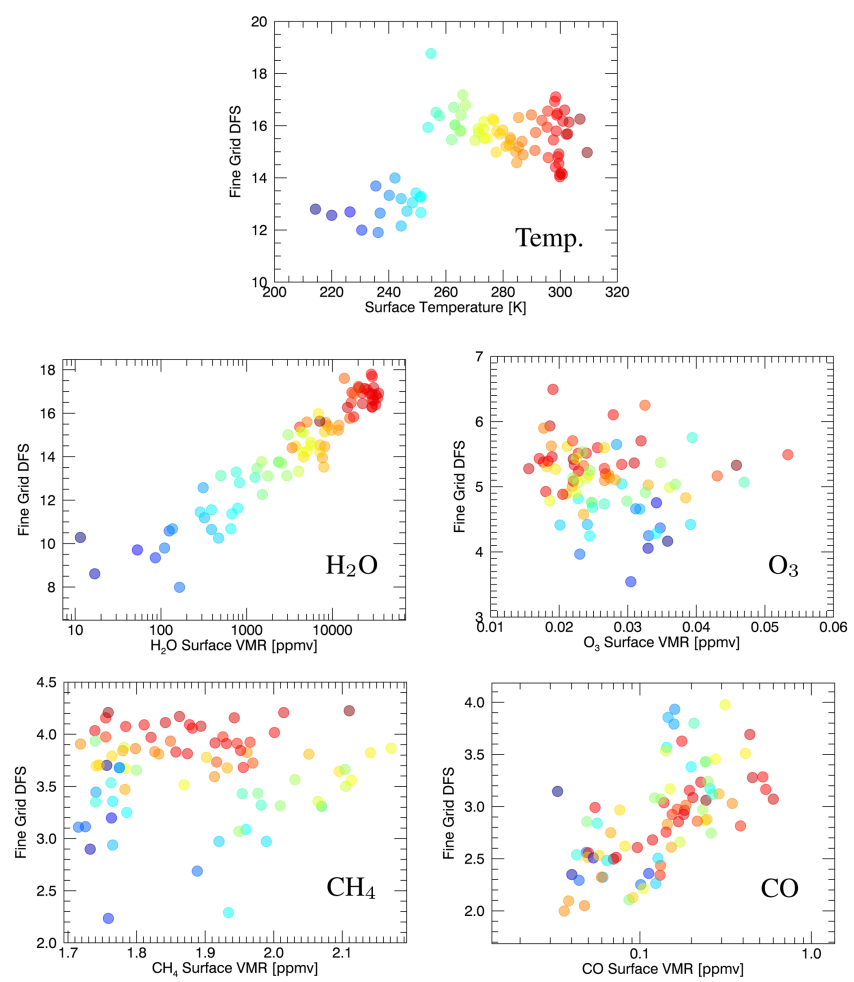

Figure 5. The DFS from the ensemble for temperature and trace gases as a function of their surface values. The points are colour coded by atmospheric surface temperature using the colour bar shown in Fig. 4.

sphere) results in a well-constrained problem, using little a priori. Once more than approximately 10 pressure levels are included in the retrieval, then adding further levels negligibly increases the DFS and introduces only a priori.

Figure 2 can be extrapolated to any trace gas or temperature profile retrieval. Including many more vertical levels than DFS adds increasingly negligible information. Therefore, a straightforward way to decide upon the number of vertical grid levels is to add a few more levels than DFS on the fine grid and check via Eq. (12) that the loss in DFS is acceptably small.

\section{Comparison of grid selection methods}

In this section the three different selection methods are compared, using a variety of atmospheric conditions for simulated IASI retrievals of temperature, $\mathrm{H}_{2} \mathrm{O}, \mathrm{O}_{3}, \mathrm{CH}_{4}$, and $\mathrm{CO}$.

\subsection{Computational details}

Radiances measured by IASI are accurately reproduced by configuring a radiative transfer model to the specifics of IASI's orbital geometry and instrument response (Ventress, 2014). Spectral modelling in this study was performed with the Reference Forward Model (RFM) using the HITRAN 2012 spectral database (Rothman et al., 2013). Jacobians, K, for gas retrievals were created for estimates of the natural logarithm of volume mixing ratio (VMR). Further information on the RFM can be found in the online manual (Dudhia, 2008).

Instrument noise was provided by the Centre National d'Etudes Spatiales (CNES) 2008 post-launch estimate (Hilton et al., 2009) and used to construct the measurement covariance matrix, $\mathbf{S}_{\epsilon}$. Cross channel correlations in $\mathbf{S}_{\epsilon}$ due to apodisation were accounted for according to Amato et al. (1998). Spectral ranges typical for IASI retrievals were selected for this study and are shown in Fig. 3. All spectral channels in the given ranges were included. Furthermore, for simplicity of analysis, joint retrievals of combined states were not considered.

\subsection{Atmospheric ensemble description}

Continuing work from the Thermodynamic Initial Guess Retrieval (TIGR) database (Chevallier et al., 2000), an ensemble of 83 atmospheres was created by Matricardi (2008) specifically for the development of the Radiative Transfer model for the Television infrared observation satellite Operational Vertical sounder (RTTOV) regression coefficients. Care was given to ensure that the atmospheric states represent the statistical variability of Earth's atmosphere, to include extreme weather events. The 83 atmospheres were originally sampled from over 120000000 profiles generated by the European Centre for Medium-Range Weather Forecasts (ECMWF) cycle30R2 forecasting system, creating sets for temperature, $\mathrm{H}_{2} \mathrm{O}$, and $\mathrm{O}_{3}$. While other trace gas profiles, such as $\mathrm{CH}_{4}$ and $\mathrm{CO}$, were generated from the Global and regional Earthsystem Monitoring using Satellite and in situ data (GEMS) database (Hollingsworth et al., 2008).

Temperature and gas profiles are presented on a fixed fine grid of 101 pressure levels ranging from 1100 to $0.005 \mathrm{hPa}$. Of the total, three atmospheres represent minimum, maximum, and mean values of the 80 independent profiles. Therefore, those three were not used in this study. Figure 4 is a visual reference showing the sampling locations and atmospheric surface temperatures for the 80 atmospheres.

The original purpose of the TIGR database was to sample representative a priori information for accurate modelling of forward or inverse radiative transfer problems. As such, a prior covariance matrix was created for each constituent studied here by calculating the sample covariance from the 80 -atmosphere ensemble. Therefore, the prior covariances used represent global statistics and include full off-diagonal 


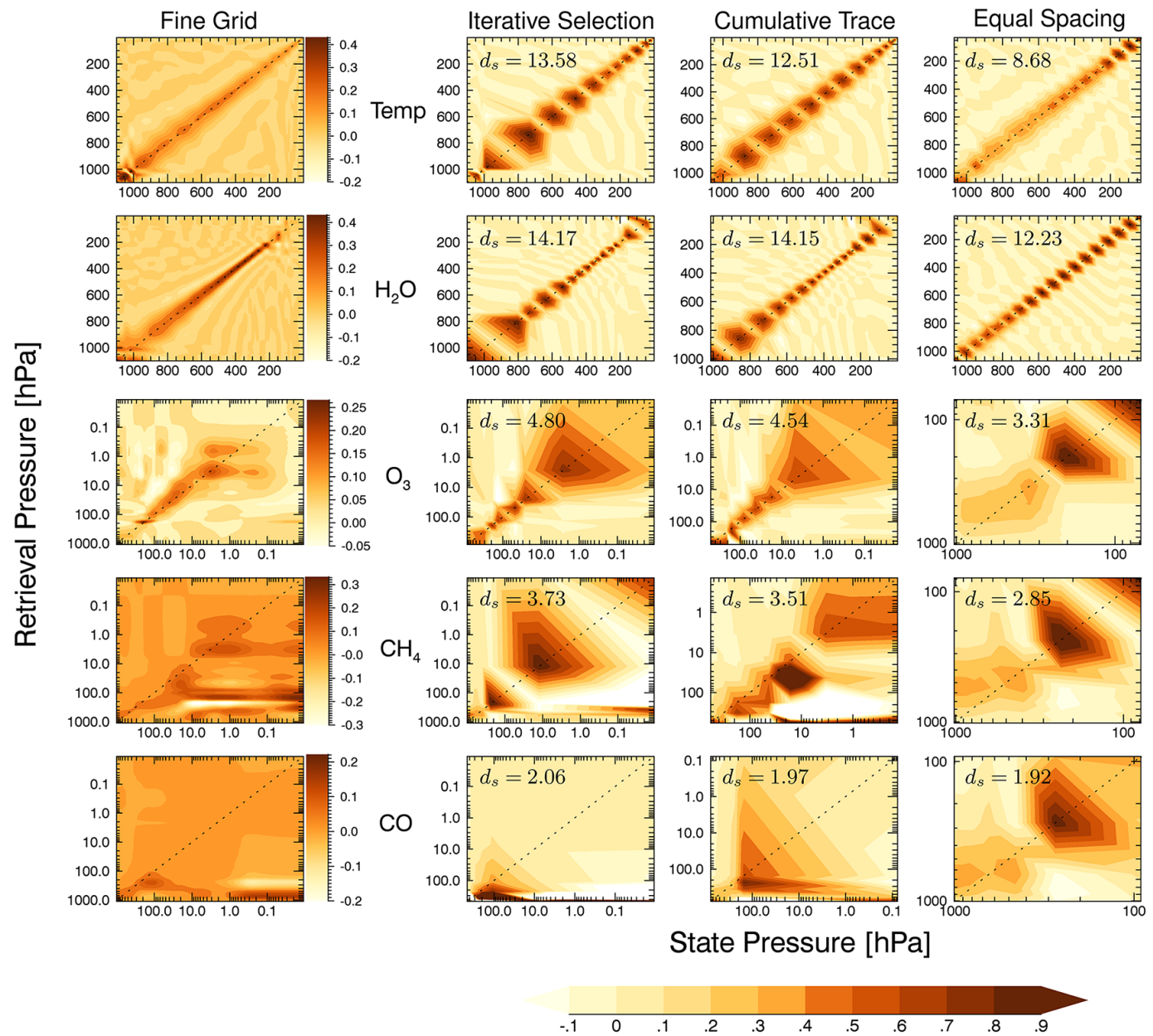

Figure 6. Contours of AKMs for a mid-latitude summer retrieval of temperature, $\mathrm{H}_{2} \mathrm{O}, \mathrm{O}_{3}, \mathrm{CH}_{4}$, and CO using IASI. Fine grid refers to AKMs evaluated on the 101 level vertical grid without interpolation from a coarser grid. The right three columns show AKMs on coarser vertical grids selected according to the methods discussed in Sects. 3.1-3.3. All three coarse grids have the same number of levels for each retrieval, chosen to be four more than the rounded DFS on the fine grid. DFS values specific to each coarse grid AKM are annotated in the top left corners. Notice that the pressure axes are specific to the AKM shown, especially the fourth column.

correlations often neglected in retrieval schemes. This choice was made primarily to highlight the effect off-diagonal sensitivities in the AKMs have upon the level selection results. While it is unlikely these particular prior covariances will be used in an operational retrieval, they may provide the most interesting case study.

Furthermore, using a global prior covariance includes greater variation than a latitude specific atmosphere may experience. Therefore, the fine grid DFS represents an upper limit to what may be achieved in an operational retrieval. Figure 5 shows the spread of DFS for each atmospheric constituent considered as a function of its surface quantity and colour coded by surface temperature. Note that the DFS of the gases vary by about a factor of 2. Additionally, there is an interesting correlation between DFS and surface temperature for $\mathrm{H}_{2} \mathrm{O}, \mathrm{O}_{3}$, and $\mathrm{CH}_{4}$ that is not apparent with $\mathrm{CO}$.

\subsection{Mid-latitude grid comparisons}

A typical mid-latitude summer atmosphere was chosen from the ensemble to help visualise the differences of selecting coarse grids from the methods in Sect. 3. Contour plots of AKMs for the mid-latitude atmosphere are shown in Fig. 6. Consider the first two rows showing AKMs for temperature and $\mathrm{H}_{2} \mathrm{O}$. On the fine grid, the retrieval responses fall mostly along the diagonal in a largely symmetric pattern. Comparing the iterative to the cumulative trace coarse grid AKMs, there are differences, but they appear slight. For the following three rows of AKMs for $\mathrm{O}_{3}, \mathrm{CH}_{4}$, and $\mathrm{CO}$, as the off-diagonal sensitivities increase the differences between coarse grids become more noticeable. In fact, these off-diagonal sensitivities are the motivating factor for using the iterative vertical selection method. Finally, AKMs for the equal spacing method are substantially different than the other two methods, be- 


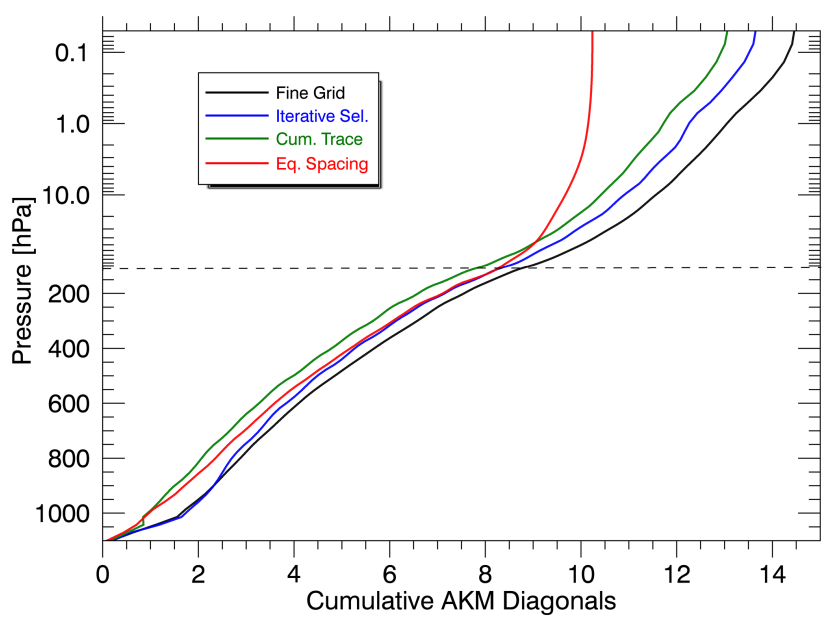

Figure 7. Cumulative diagonals of the AKM for a temperature retrieval on the 101 level fine grid as well as 18 level coarse grid AKMs (Sects. 3.1-3.3) interpolated to the fine grid.

cause the location of information content, particularly in the stratosphere, is completely ignored.

These off-diagonal responses in the fine grid AKMs for $\mathrm{O}_{3}, \mathrm{CH}_{4}$, and $\mathrm{CO}$ are attributed to both spectral correlations from $\mathbf{K}^{\mathrm{T}} \mathbf{S}_{\epsilon}^{-1} \mathbf{K}$ and the off-diagonal terms in $\mathbf{S}_{\mathrm{a}}$. If the full sample covariance were replaced with a simpler tridiagonal matrix for $\mathbf{S}_{\mathrm{a}}$ (i.e. smoothness constraint) or just a diagonal matrix, then the fine grid AKMs become more diagonal and symmetric. However, there are still spectral correlations to consider. In this case, the iterative vertical selection method and the cumulative trace of $\mathbf{A}$ method will produce different grids.

Figure 7 shows the cumulative diagonal of the interpolated AKMs for each coarse grid method compared to the fine grid AKM for a temperature retrieval. Notice that creating a coarse grid from equal spacings of pressure reduces stratospheric DFS by approximately four. The cumulative trace coarse grid maintains greater DFS, but shows an immediate decrease in DFS between the surface and $800 \mathrm{hPa}$. This is because the morphology of the AKM changes as reducing vertical levels bins off-diagonal elements into the new diagonal components on the coarser grid. The iterative method grid keeps the greatest DFS along the profile compared to the other two methods, because it accounts for off-diagonal responses during the selection process. The next section discusses if the differences are significant to justify the more complex iterative selection method.

\subsection{Ensemble comparisons}

Vertical selection comparisons were made using the same number of vertical levels specific to each atmosphere to isolate differences solely due to the method. The selection method that retains the greatest DFS on average for the 80 atmospheres is the iterative method from Sect. 3.3.

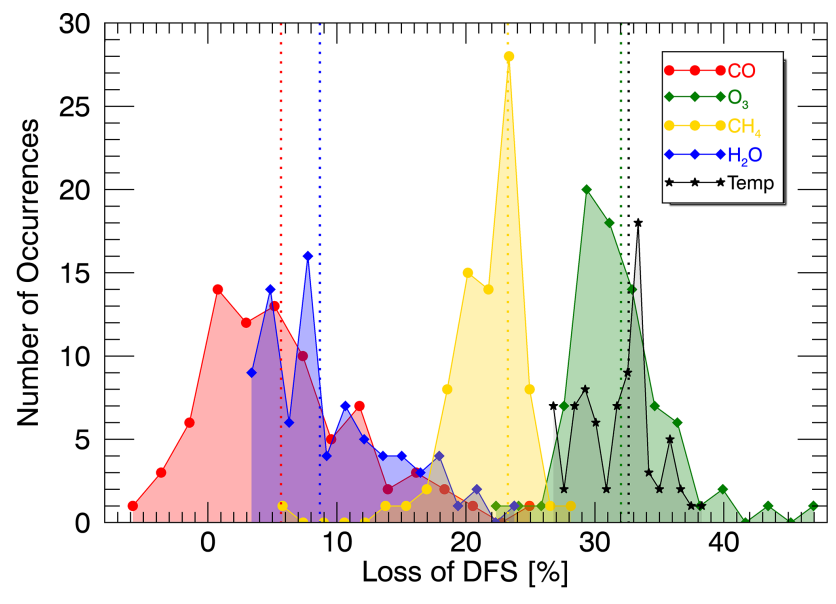

Figure 8. Histograms of 15 equally spaced bins showing the percent loss in DFS by using a coarse vertical grid with equal pressure spacing vs. the coarse grid from the iterative method. The dotted vertical lines show the median values of the 80 -atmosphere distribution.

Figure 8 shows histograms of the loss in DFS by using an equally spaced vertical pressure grid as compared to the iterative method. For the majority of atmospheres the difference in DFS for $\mathrm{CO}$ and $\mathrm{H}_{2} \mathrm{O}$ is less than $10 \%$. In fact, there are 10 atmospheres for a $\mathrm{CO}$ retrieval in which the equal spacing grid provided more DFS than the iterative grid, which highlights the fact that the iterative method does not guarantee finding the global maximum of DFS. However, the majority of atmospheres with equal spacing reduce the DFS by over $20 \%$ for $\mathrm{CH}_{4}$ and over $30 \%$ for $\mathrm{O}_{3}$ and temperature. This significant loss in DFS results from ignoring stratospheric information content. While using equal spacings in pressure seems like an obviously poor idea for retrieving $\mathrm{O}_{3}$, there is also significant stratospheric information for $\mathrm{CH}_{4}$ and temperature that should be considered.

The loss of DFS by using a vertical grid selected from the cumulative diagonal of A compared to the iterative method is shown in Fig. 9. For the majority of temperature retrievals the loss in DFS is less than $10 \%$, while the loss for trace gases is less than $5 \%$. This shows that the benefit from considering off-diagonal sensitivities in the selection process is slight, especially for the trace gases considered.

\section{Globally designed grid}

Finally, when constructing a retrieval algorithm with IASI or another infrared sounding instrument, the developer may consider whether to use a constant vertical grid or one that optimally adapts to the observed atmosphere based on the prior state and converging profile. Figure 10 are histograms showing the loss of DFS when using a constant vertical grid optimised globally vs. an atmosphere specific grid. The global vertical grid was constructed following the iterative method, but considering an average DFS from a tropical, 


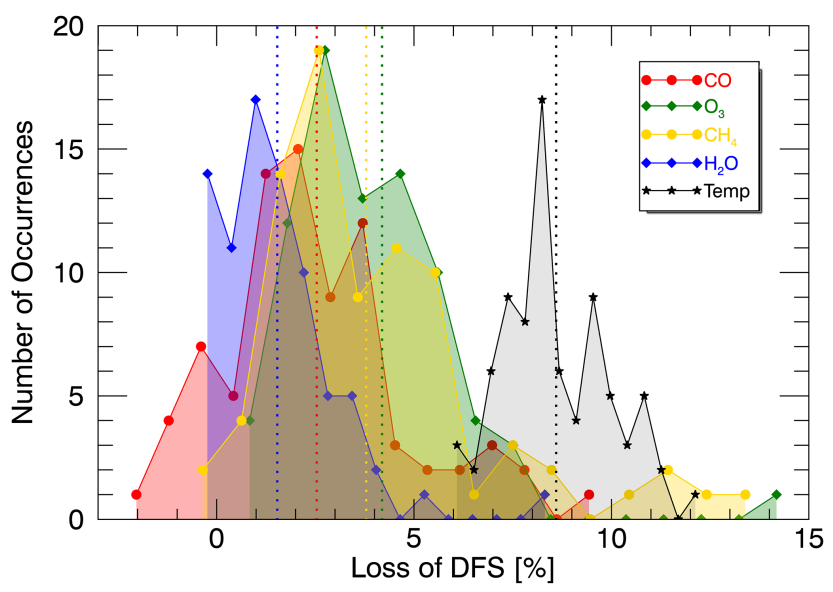

Figure 9. Histograms of 15 equally spaced bins showing the percent loss in DFS by creating a coarse vertical grid from the cumulative trace of $\mathbf{A}$ vs. the coarse grid from the iterative method. The dotted vertical lines show the median values of the 80 -atmosphere distribution.

Table 1. Summaries of the ensemble results from Figs. 8-10. The second column shows the ensemble mean DFS for each retrieval using the iterative method. The last three columns show the median percent loss of DFS for the histograms displayed.

\begin{tabular}{lrrrr}
\hline Retrieval & $\overline{d_{\mathrm{s}}}$ & $\begin{array}{r}\text { Eq PRE } \\
{[\% \text { loss }]}\end{array}$ & $\begin{array}{r}\operatorname{Diag}(\mathbf{A}) \\
{[\% \text { loss }]}\end{array}$ & $\begin{array}{r}\text { Global } \\
{[\% \text { loss }]}\end{array}$ \\
\hline Temperature & 15.7 & 32.6 & 8.6 & 12.1 \\
$\mathrm{H}_{2} \mathrm{O}$ & 13.8 & 8.7 & 1.5 & 12.1 \\
$\mathrm{O}_{3}$ & 5.1 & 32.0 & 4.2 & 2.5 \\
$\mathrm{CH}_{4}$ & 3.8 & 23.1 & 3.8 & 2.1 \\
$\mathrm{CO}$ & 2.9 & 5.8 & 2.5 & 2.3 \\
\hline
\end{tabular}

mid-latitude, polar summer, and polar winter atmosphere as the metric of quality.

Notice that the median DFS losses are less than $3 \%$ for $\mathrm{O}_{3}, \mathrm{CH}_{4}$, and $\mathrm{CO}$. However, there are long tails extending past $10 \%$ for the more extreme atmospheres. Additionally, temperature and $\mathrm{H}_{2} \mathrm{O}$ show DFS loss values between 10 and $20 \%$, suggesting that the vertical location of information varies more significantly than the other gases considered. Therefore, an adaptive atmosphere specific vertical grid may improve retrievals of temperature and water vapour from an information perspective. However, the practicalities of implementing an adaptive grid may make this increase in DFS an undesirable trade-off when attempting to produce time averaged or cross-platform analyses.

To summarise Figs. 8-10, the ensemble median values are shown in Table 1 along with the mean DFS values for the referenced iterative selection method.

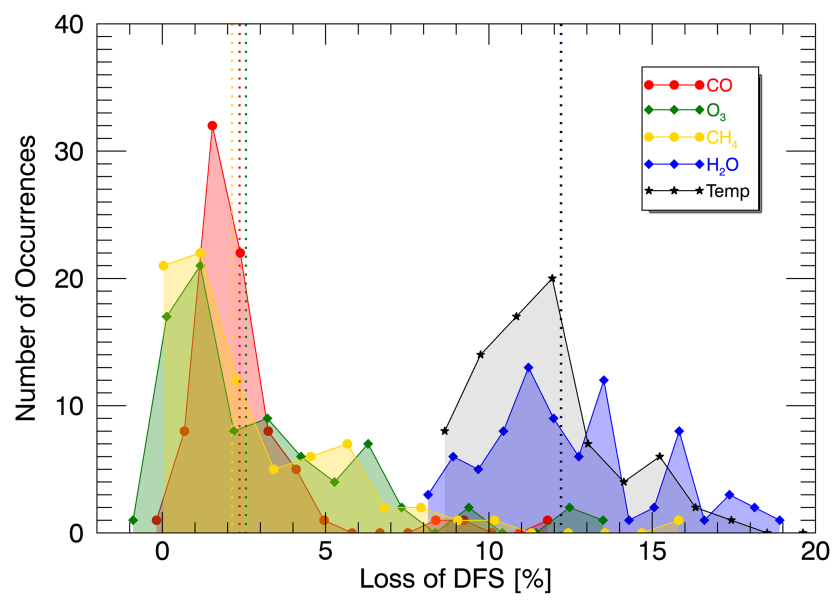

Figure 10. Histograms of 15 equally spaced bins showing the loss in DFS by using a constant globally optimised vertical grid vs. an atmosphere specific grid. The dotted vertical lines show median values of the 80-atmosphere distribution, where the temperature and $\mathrm{H}_{2} \mathrm{O}$ medians overlay each other.

\section{Conclusions}

When retrieving atmospheric profiles of temperature and trace gases from infrared spectral radiances, it is important to consider where in the vertical profile the estimates are made. A new iterative method for selecting a vertical grid was proposed and shown to outperform previously used selection methods by accounting for correlations and sensitivities between different vertical levels. Other compared methods of establishing a vertical grid coarser than the radiative transfer grid were using levels equally spaced in pressure and selecting levels by interpolating along the cumulative diagonal of the fine grid AKM.

The 80-atmosphere ensemble created to parametrise RTTOV was used to systematically compare the different vertical grid selection methods for temperature, $\mathrm{H}_{2} \mathrm{O}, \mathrm{O}_{3}, \mathrm{CH}_{4}$, and $\mathrm{CO}$. For the majority of atmospheres, using a vertical grid with equal pressure spacings resulted in a $20-40 \%$ loss of DFS for temperature, $\mathrm{O}_{3}$, and $\mathrm{CH}_{4}$. Median DFS losses for $\mathrm{H}_{2} \mathrm{O}$ and $\mathrm{CO}$ were less than $10 \%$. In general, this shows that a significant proportion of DFS can be retained by considering the vertical location of the information content as opposed to choosing a vertical grid based on convenience.

Comparing to the cumulative diagonal of $\mathbf{A}$ method shows that greater DFS can be achieved with the iterative method, but median losses are less than $5 \%$ for the trace gases and less than $10 \%$ for temperature retrievals. This slight reduction in DFS is unlikely to affect the quality of retrievals in a noticeable way for the majority of atmospheric cases. Therefore, the simpler and less expensive method of interpolating along the cumulative diagonal of $\mathbf{A}$ will likely be sufficient, except for possibly extreme atmospheric scenarios with high inter-level correlations. 
Finally, much effort is spent making retrieval schemes run faster. Naturally, one would prefer to design a coarse vertical grid just once and apply that to all scenarios, rather than optimise a grid for each retrieved atmosphere. This depends upon the variability of vertical information content. The median loss of DFS for $\mathrm{O}_{3}, \mathrm{CH}_{4}$, and $\mathrm{CO}$ when using a globally optimised grid vs. an atmosphere specific grid was less than $3 \%$. For the majority of atmospheres, using a static grid results in a negligible retrieval difference for these three gases. However, the loss of DFS for temperature and $\mathrm{H}_{2} \mathrm{O}$ is more appreciable, greater than $10 \%$, implying the location of vertical information is more variable than the other trace gases considered. Whether or not to account for information variability with temperature and $\mathrm{H}_{2} \mathrm{O}$ ultimately depends upon the motivation for computation speed and tolerance of the DFS reductions presented.

Acknowledgements. Portions of this work were funded by the United States Air Force. The views expressed in this article are those of the author and do not reflect the official policy or position of the United States Air Force, Department of Defense or the US Government.

Edited by: A. Butz

\section{References}

Amato, U., De Canditiis, D., and Serio, C.: Effect of apodization on the retrieval of geophysical parameters from Fourier-transform spectrometers, Appl. Optics, 37, 6537-6543, 1998.

August, T., Klaes, D., Schlüssel, P., Hultberg, T., Crapeau, M., Arriaga, A., O'Carroll, A., Coppens, D., Munro, R., and Calbet, X.: IASI on Metop-A: operational level 2 retrievals after five years in orbit, J. Quant. Spectrosc. Ra., 113, 1340-1371, 2012.

Aumann, H., Chahine, M., Gautier, C., Goldberg, M., Kalnay, E., McMillin, L., Revercomb, H., Rosenkranz, P., Smith, W., Staelin, D., Strow, L., and Susskind, J.: AIRS/AMSU/HSB on the Aqua mission: Design, science objectives, data products, and processing systems, IEEE T. Geosci. Remote, 41, 253-264, 2003.

Bey, I., Jacob, D. J., Yantosca, R. M., Logan, J. A., Field, B. D., Fiore, A. M., Li, Q., Liu, H. Y., Mickley, L. J., and Schultz, M. G.: Global modeling of tropospheric chemistry with assimilated meteorology: model description and evaluation, J. Geophys. Res.-Atmos., 106, 23073-23095, 2001.

Bowman, K. W., Rodgers, C. D., Kulawik, S. S., Worden, J., Sarkissian, E., Osterman, G., Steck, T., Lou, M., Eldering, A., Shephard, M., Brown, P., Rinsland, C., Gunson, M., and Beer, R.: Tropospheric emission spectrometer: retrieval method and error analysis, IEEE T. Geosci. Remote, 44, 1297-1307, 2006.
Brasseur, G., Hauglustaine, D., Walters, S., Rasch, P., Müller, J.-F., Granier, C., and Tie, X.: MOZART, a global chemical transport model for ozone and related chemical tracers: 1. Model description, J. Geophys. Res.-Atmos., 103, 28265-28289, 1998.

Ceccherini, S., Raspollini, P., and Carli, B.: Optimal use of the information provided by indirect measurements of atmospheric vertical profiles, Opt. Express, 17, 4944-4958, 2009.

Chevallier, F., Chédin, A., Chéruy, F., and Morcrette, J.-J.: TIGRlike atmospheric-profile databases for accurate radiative-flux computation, Q. J. Roy. Meteor. Soc., 126, 777-785, 2000.

Dudhia, A.: Reference Forward Model (RFM), available at: http: //www.atm.ox.ac.uk/RFM (last access: January 2015), 2008.

Han, Y., Revercomb, H., Cromp, M., Gu, D., Johnson, D., Mooney, D., Scott, D., Strow, L., Bingham, G., Borg, L., Chen, Y., DeSlover, D., Esplin, M., Hagan, D., Jin, X., Knuteson, R., Motteler, H., Predina, J., Suwinski, L., Taylor, J., Tobin, D., Tremblay, D., Wang, C., Wang, L., Wang, L., and Zavyalov, V.: Suomi NPP CrIS measurements, sensor data record algorithm, calibration and validation activities, and record data quality, J. Geophys. Res.-Atmos., 118, 12734-12748, 2013.

Hilton, F., Atkinson, N., English, S., and Eyre, J.: Assimilation of IASI at the Met Office and assessment of its impact through observing system experiments, Q. J. Roy. Meteor. Soc., 135, 495$505,2009$.

Hollingsworth, A., Engelen, R., Benedetti, A., Dethof, A., Flemming, J., Kaiser, J., Morcrette, J., Simmons, A., Textor, C., Boucher, O., Chevallier, F., Rayner, P., Elbern, H., Eskes, H., Granier, C., Peuch, V.-H., Rouil, L., and Schultz, M. G.: Toward a monitoring and forecasting system for atmospheric composition: the GEMS project, B. Am. Meteorol. Soc., 89, 1147-1164, 2008.

Joiner, J. and Da Silva, A.: Efficient methods to assimilate remotely sensed data based on information content, Q. J. Roy. Meteor. Soc., 124, 1669-1694, 1998.

Kulawik, S. S., Osterman, G., Jones, D. B., and Bowman, K. W.: Calculation of altitude-dependent Tikhonov constraints for TES nadir retrievals, IEEE T. Geosci. Remote, 44, 1334-1342, 2006.

Matricardi, M.: The generation of RTTOV regression coefficients for IASI and AIRS using a new profile training set and a new line-by-line database, ECMWF Research Dept, Reading, UK, Tech. rep., Tech. Memo., 564, available at: http://www.ecmwf. int/publications (last access: February 2015), 2008.

Payne, V. H., Clough, S. A., Shephard, M. W., Nassar, R., and Logan, J. A.: Information-centered representation of retrievals with limited degrees of freedom for signal: application to methane from the Tropospheric Emission Spectrometer, J. Geophys. Res., 114, D10307, doi:10.1029/2008JD010155, 2009.

Rodgers, C. D.: Inverse Methods for Atmospheric Sounding: Theory and Practice, vol. 2, World Scientific, Singapore, 2000.

Rothman, L., Gordon, I., Babikov, Y., et al.: The HITRAN2012 molecular spectroscopic database, J. Quant. Spectrosc. Ra., 130, 4-50, 2013.

Ventress, L.: Atmospheric sounding using IASI, PhD thesis, Oxford University, Oxford, 2014. 
Ventress, L. and Dudhia, A.: Improving the selection of IASI channels for use in numerical weather prediction, Q. J. Roy. Meteor. Soc., 140, 2111-2118, doi:10.1002/qj.2280, 2013.

von Clarmann, T.: Smoothing error pitfalls, Atmos. Meas. Tech., 7, 3023-3034, doi:10.5194/amt-7-3023-2014, 2014.

von Clarmann, T. and Grabowski, U.: Elimination of hidden a priori information from remotely sensed profile data, Atmos. Chem. Phys., 7, 397-408, doi:10.5194/acp-7-397-2007, 2007.
Worden, J., Bowman, K., Noone, D., Beer, R., Clough, S., Eldering, A., Fisher, B., Goldman, A., Gunson, M., Herman, R., Kulawik, S. S., Lampel, M., Luo, M., Osterman, G., Rinsland, C., Rodgers, C., Sander, S., Shephard, M., and Worden, H.: Tropospheric Emission Spectrometer observations of the tropospheric $\mathrm{HDO} / \mathrm{H}_{2} \mathrm{O}$ ratio: Estimation approach and characterization, J. Geophys. Res., 111, D16309, doi:10.1029/2005JD006606, 2006. 